\title{
Erratum to: Pro-Cognitive Properties of the Immunomodulatory Polypeptide Complex, Yolkin, from Chicken Egg Yolk and Colostrum-Derived Substances: Analyses Based on Animal Model of Age-Related Cognitive Deficits
}

\author{
Marta Lemieszewska $^{1}$ - Marta Jakubik-Witkowska ${ }^{1} \cdot$ Bartłomiej Stańczykiewicz $^{1}$ \\ Aleksandra Zambrowicz ${ }^{2}$ - Agnieszka Zabłocka ${ }^{3}$ - Antoni Polanowski ${ }^{2}$. \\ Tadeusz Trziszka ${ }^{2} \cdot J_{0 a n n a}$ Rymaszewska ${ }^{1}$ \\ Published online: 9 May 2016 \\ (C) L. Hirszfeld Institute of Immunology and Experimental Therapy, Wroclaw, Poland 2016
}

\section{Erratum to: Arch. Immunol. Ther. Exp. DOI 10.1007/s00005-016-0392-z}

The author would like to correct the following errors in the online publication of the article:

In page 1, under the Abstract section, line 14 should read as "Two different doses of the studied specimens based on previous comparative studies and two different routes of administration (oral and intraperitoneal) were used".

Under the Introduction heading, line 3 should read as "Cognitive disorders affecting memory and learning abilities normally occur in the process of aging, mild cognitive impairment and in Alzheimer's disease (Lindeboom and Weinstein 2004; Pepeu 2004)."

Under the Introduction heading, line 32 should read as "Positive results of preliminary clinical trials on patients with Alzheimer's disease, in which from a total of 15 patients receiving oral CLN at a dose of $100 \mu \mathrm{g}$, eight improved according to the MiniMental State Examination, while disease symptoms stabilized in the remaining seven patients (Leszek et al. 1999)."

The corrected Keywords section should read as follows:

Keywords Immunomodulatory peptides - Chicken egg yolk $\cdot$ Colostrinin $\cdot$ Coloco $\cdot$ Learning $\cdot$ Memory $\cdot$ Dementia Age-related disorders · Cognitive deficits · Yolkin

The online version of the original article can be found under doi:10.1007/s00005-016-0392-z.

Marta Lemieszewska

m.lemieszewska@gmail.com

1 Division of Consultation Psychiatry and Neuroscience, Department of Psychiatry, Wroclaw Medical University, 10 Pasteura Street, 50-367 Wroclaw, Poland

2 Department of Animal Products Technology and Quality Management, Wroclaw University of Environmental and Life Sciences, 37 Chelmonskiego Str, 51-630 Wroclaw, Poland

3 Department of Immunochemistry, Ludwik Hirszfeld Institute of Immunology and Experimental Therapy, Polish Academy of Sciences, 12 Weigla Str, 53-114 Wroclaw, Poland 